\title{
Hendrik Kasten und Denis Vogel: Grundlagen der ebenen Geometrie - Eine zugängliche aber exakte Einführung in die ebene Geometrie
}

\author{
Springer Spektrum 2019, IX + 333 Seiten, \\ ISBN 978-3-662-57620-5, €32,99; eBook ISBN 978-3-662-57621-2, \\ $€ 24,99$
}

\section{Max Hoffmann}

Angenommen: 23. März 2021 / Online publiziert: 6. Mai 2021

(C) Der/die Autor(en) 2021

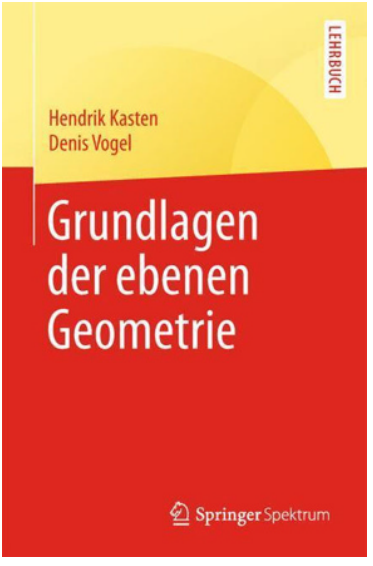

Der Titel „Grundlagen der ebenen Geometrie“ allein lässt wenig Rückschlüsse auf die Ausrichtung des Buches zu. Deswegen möchte ich zu Beginn einige generelle Einordnungen vornehmen. Das Buch beruht ausweislich des Vorworts auf einem Vorlesungsskript der Autoren. Die Veranstaltung trägt den Titel „Einführung in die Geometrie" und setzt die Inhalte der Grundvorlesungen zur Analysis und Linearen Algebra voraus. Das Buch liefert eine Zusammenstellung von Standardthemen der ebenen Geometrie, sowohl aus axiomatischer Sicht als auch aus Perspektive der analytischen Geometrie. Dabei setzten es sich die Autoren zum Ziel, beide Zugänge als miteinander verbunden darzustellen. Auch wenn es ein Kapitel zur nichteuklidischen Geometrie (meint hier: hyperbolisch und elliptisch) gibt, liegt der Schwerpunkt des Buches im euklidischen.

Die vorgeschaltete Einleitung enthält eine kurze Reise durch die Geschichte der ebenen Geometrie mit Haltestellen bei Euklid, Hilbert und Descartes. Das erste inhaltliche Kapitel (Kapitel 2) trägt den Titel „Inzidenzgeometrie“. Aufbauend auf Inzidenzebenen werden dort affine und projektive Ebenen (inkl. des Dualitätskonzepts) eingeführt und verglichen sowie abschließend die einschlägigen Schnittpunktsätze

\footnotetext{
M. Hoffmann ( $\square)$

Universität Paderborn, Paderborn, Deutschland

E-Mail: max.hoffmann@math.upb.de

Pelizaeus-Gymnasium Paderborn, Paderborn, Deutschland
} 
behandelt. Kapitel 3 („Hilbertebenen“) ist im Wesentlichen eine Präsentation von Hilberts Festschrift zu den Grundlagen der Geometrie. Dabei wird en passant gezeigt, unter welchen Bedingungen die vorher eingeführten Inzidenzebenen Modelle für einzelne Teilmengen der Hilbert-Axiomatik sind. Das anschließende Kapitel 4 (,Der Hauptsatz“) trägt in seinem Titel die Referenz auf die zentrale Isomorphie von axiomatisch beschriebenen euklidischen Ebenen und der euklidischen Standardebene im Sinne der analytischen Geometrie. Dieser Hauptsatz wird von den Autoren dabei unter dem Credo vorgestellt, dass er es erlaubt, Aussagen der euklidischen Geometrie sowohl mit synthetischen als auch mit analytischen Mitteln zu beweisen, ohne dass das Argument an Allgemeingültigkeit verliert. In Kapitel 5 werden dann eine Vielzahl an Standard-Sätzen der euklidischen Geometrie unter Verwendung der in den vorherigen Kapiteln bereitgestellen Werkzeuge bewiesen. Die beiden letzten Kapitel des Buches zeigen noch einmal zwei andere Perspektiven auf ebene Geometrie: Kapitel 6 beschäftigt sich mit geometrischen Konstruktionen (Zirkel und/ oder Lineal) und Kapitel 7 mit nichteuklidischer Geometrie (hyperbolische Ebenen und elliptische Geometrie).

Alle Ausführungen sind durchgehend von hoher Präzision und werden durch viele instruktive Zeichnungen unterstützt. Die Präzision wird dabei unter anderem durch einen konsequent eingesetzten Formalismus unterstützt. Ich schätze und nutze das Buch als Nachschlagewerk für geometrische Themen, die mir als Lehrender in der Lehrerausbildung und als Geometriedidaktiker begegnen. Bemerkenswert ist hier die reichhaltige und synergetische Zusammenstellung verschiedener Perspektiven auf ebene Geometrie. Damit unterscheidet sich das Buch von anderen Büchern zur ebenen Geometrie, die oft entweder sehr überblicks- und spotlightartig vorgehen oder zwar sehr präzise sind, aber dabei auch nur in einem kleinen inhaltlichen Feld bleiben.

Auf dem Klappentext wird die Zielgruppe des Buches als „Studierende der universitären Mathematikstudiengänge“" sowie Gymnasiallehrkräfte angegeben. Ich habe allerdings Zweifel, ob das Buch den Belangen dieser beiden Zielgruppen gerecht wird. Für die Empfehlung als Studierendenlektüre fehlen mir persönlich einordnende und reflektierende Textbausteine, die Lernende an die Hand nehmen, sie auf besonders wichtige oder schöne Dinge aufmerksam machen und Aktuelles in Relation zu dem bereits Behandelten aber auch in Relation zu den kommenden Inhalten setzen. Für den geometrisch nicht vorgebildeten Leser fehlt es an Schwerpunktsetzungen und den Anknüpfungspunkten zu bekanntem Vorwissen (sowohl zur Schulgeometrie als auch zu anderen Veranstaltungen). Das oben bereits erwähnte Zusammenspiel von synthetischen und analytischen Argumenten wird zwar vollzogen jedoch nicht reflektiert. Hilfreich wären beispielweise Gegenüberstellungen und vergleichende Analysen beider Argumentationsvarianten zum selben Theorem.

Für die Verwendung durch Lehrkräfte fehlt mir die explizite Diskussion von Bezugspunkten zur Schulmathematik, die einer Lehrkraft helfen, beim „Nachschlagen“ einen Einstiegspunkt zu finden, ohne das Buch systematisch durcharbeiten zu müssen. Wichtige Begriffe der ebenen Schulgeometrie, wie „Symmetrie“ kommen gar nicht vor. Darüber hinaus führt die enge Symboldichte und die Verwendung einer Vielzahl von Nichtstandardnotationen dazu, dass ein Einstieg mitten im Buch (wie er beim Nachschlagen oft vorkommt) immer mit einem großen notationellen Orien- 
tierungsaufwand einher geht. Das aus Schulsicht hochgradig relevante Thema der geometrischen Konstruktionen geht meiner Einschätzung nach gerade durch einen anspruchsvollen und abstrakten Zugang an dieser Zielgruppe vorbei.

„Grundlagen der ebenen Geometrie“ von Hendrik Kasten und Denis Vogel ist eine präzise, reichhaltige und sorgfältig aufgebaute Zusammenstellung von wichtigen Aspekten der ebenen Geometrie. Wem sich die Schönheit der Geometrie bereits an anderer Stelle offenbart hat, der wird mit diesem Buch ein wirklich instruktives Nachschlage- und Vertiefungswerk finden. Die Beschäftigung mit klassischer Geometrie ist, gerade in den Nicht-Lehramts-Studiengängen, leider von immer geringerer Bedeutung. Ich befürchte allerdings, dass hier für Geometrie-Novizinnen und Novizen, die Leichtigkeit und Schönheit geometrischer Argumente auf der Strecke bleibt. Für meinen Geschmack wird die Geometrie ein wenig zu sehr abgearbeitet und dabei zu wenig zelebriert.

Funding Open Access funding enabled and organized by Projekt DEAL.

Open Access Dieser Artikel wird unter der Creative Commons Namensnennung 4.0 International Lizenz veröffentlicht, welche die Nutzung, Vervielfältigung, Bearbeitung, Verbreitung und Wiedergabe in jeglichem Medium und Format erlaubt, sofern Sie den/die ursprünglichen Autor(en) und die Quelle ordnungsgemäß nennen, einen Link zur Creative Commons Lizenz beifügen und angeben, ob Änderungen vorgenommen wurden.

Die in diesem Artikel enthaltenen Bilder und sonstiges Drittmaterial unterliegen ebenfalls der genannten Creative Commons Lizenz, sofern sich aus der Abbildungslegende nichts anderes ergibt. Sofern das betreffende Material nicht unter der genannten Creative Commons Lizenz steht und die betreffende Handlung nicht nach gesetzlichen Vorschriften erlaubt ist, ist für die oben aufgeführten Weiterverwendungen des Materials die Einwilligung des jeweiligen Rechteinhabers einzuholen.

Weitere Details zur Lizenz entnehmen Sie bitte der Lizenzinformation auf http://creativecommons.org/ licenses/by/4.0/deed.de. 